\title{
Investigations on the antifatigue and antihypoxic effects of Paecilomyces hepiali extract
}

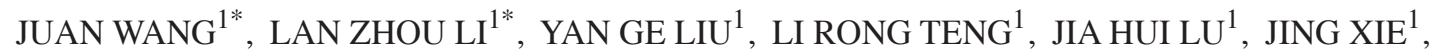 \\ WEN JI HU ${ }^{1}$, YAN LIU ${ }^{1}$, YANG LIU ${ }^{2}$, DI WANG ${ }^{1,3}$ and LE SHENG TENG ${ }^{1,3}$ \\ ${ }^{1}$ School of Life Sciences, Jilin University, Changchun, Jilin 130012; \\ ${ }^{2}$ Engineering Research Center of Chinese Ministry of Education for Edible and Medicinal Fungi, \\ Jilin Agricultural University, Changchun, Jilin 130118; ${ }^{3}$ The State Engineering Laboratory of AIDS Vaccine, \\ Jilin University, Changchun, Jilin 130012, P.R. China
}

Received February 6, 2015; Accepted December 2, 2015

DOI: $10.3892 / \mathrm{mmr} .2015 .4734$

\begin{abstract}
Paecilomyces hepiali, one of the most valuable and effective Chinese medicinal herbs, possesses potential antioxidant, immunomodulatory, antitumor and anti-inflammatory properties. The present study aimed to investigate the antifatigue and antihypoxic effects of Paecilomyces hepiali extract (PHC) in a mouse model. Using a rotating rod, forced swimming and running assessment, the antifatigue activity of PHC was determined. PHC administration for 7 days had no effect on mouse horizontal or vertical movement, indicating no neurotoxicity at the selected doses was observed. Using a normobaric hypoxia, sodium nitrite toxicosis and acute cerebral ischemia assessments, $\mathrm{PHC}$ was confirmed to possess antihypoxic effects. PHC treatment for 7 days significantly enhanced the serum and liver levels of adenosine triphosphate, superoxide dismutase and glutathione peroxidase, prior to and following $60 \mathrm{~min}$ of swimming. The levels of antioxidant-associated proteins in the livers of the mice were analyzed using western blotting. PHC effectively increased the expression levels of phosphorylated (p)-5'-monophosphate (AMP)-activated protein kinase (AMPK), p-protein kinase B (AKT) and p-mammalian target of rapamycin (mTOR). The results of the present study demonstrated that PHC efficiently enhanced endurance from fatigue and had antihypoxic effects through elevation of the antioxidant capacity in the serum and liver, at least in part through the AMPK and AKT/mTOR pathways. These results indicate the potential of this natural
\end{abstract}

Correspondence to: Mr. Di Wang, School of Life Sciences, Jilin University, 2699 Qianjin Street, Changchun, Jilin 130012, P.R. China

E-mail: jluwangdi@gmail.com

*Contributed equally

Key words: Paecilomyces hepiali, antifatigue, 5'-adenosine monophosphate-activated protein kinase, antihypoxia, protein kinase $\mathrm{B} /$ mammaliam target of rapamycin product as an antioxidant in the treatment of fatigue, hypoxia and their associated diseases.

\section{Introduction}

Cordyceps sinensis, a Chinese caterpillar fungus, is known to be one of the most valuable and effective Chinese medicinal herbs, which possesses potential antioxidant, immunomodulatory, antitumor and anti-inflammatory properties (1). Paecilomyces hepiali, a parasitic fungus generally found in Cordyceps sinensis, contains similar chemical constituents and exhibits similar bioactivities (2). Polysaccharide-enriched extract, separated from Paecilomyces hepiali (PHC), has been reported as the major active element, which exhibit anti-oxidant activity (3), limit A549 cell proliferation and induce apoptosis (4). In our previous experiments, Cordyceps militaris polysaccharides were confirmed to possess antidiabetic, antinephropathic and antihypoxic activities $(5,6)$. However, the antifatigue and antihypoxic effects of Paecilomyces hepiali mycelium remain to be elucidated.

Fatigue is characterized by a physical and/or mental weariness, which results in negative effects on work performance and exercise intensity, family life and social relationships (7). Physical fatigue, a complex condition, is described as a time-dependent, exercise-induced reduction in the maximal force-generating capacity of a muscle (8). Intense exercise results in the accumulation of reactive free radicals and leads to the consumption of adenosine triphosphate (ATP) and glycogen (9). Energy metabolism is involved in the pathophysiology of fatigue, and hypoxia, which occurs during acute and chronic vascular disease, cancer and stroke, is defined as a decrease in the normal level of tissue oxygen tension (10). As reported previously, hypoxia is also associated with energy metabolism (11). 5'-AMP-activated protein kinase (AMPK) is a key regulator of cellular and whole body energy balance (12). It acts to suppress anabolic ATP-consumption pathways, and stimulates catabolic ATP-generating pathways (13). In addition, the antioxidant enzyme system protects against excessive or exhaustive exercise-induced oxidative damage, and is associated with physical status in athletes (14). Enhanced antioxidant enzyme activity prolongs exercise performance, and 
reduces physical fatigue and hypoxia $(15,16)$. The identification of natural antioxidants originating from plants has been an area of increased attention (17).

Pharmacological drugs or therapies used for treating fatigue and hypoxia remain unsatisfactory to meet individual requirements effectively. Additionally, the majority of the broad-spectrum drugs exhibit adverse effects (18). Delaying the occurrence of fatigue and hypoxia, and promoting rapid recovery are current foci of medical investigations (7). The prevalence of potential alternative medicines derived from herbs have been increasing worldwide, which can be used not only for medicinal purposes, but also for food preservation, as dietary supplements or functional foods, and in cosmetics (17). Herba rhodiolae, a traditional Chinese herb, is commonly used by the Tibetan population for the treatment of hypoxia (19), which also leads to the enhancement of fatigue-associated movements and levels of key metabolites of glycolysis, including ATP (20). Based on previous evidence, the present study hypothesized that PHC-enriched extraction may possess antifatigue and antihypoxic activities. To confirm this hypothesis, the present study aimed to investigate the associated biological activities of Paecilomyces hepiali using a mouse model. In addition, ATP metabolism and antioxidant enzyme activities were detected in the serum and liver tissues. To further analyze its underlying mechanism, the phosphorylation of protein kinase B (AKT), mammalian target of rapamycin (mTOR) and AMPK in liver were determined via western blot analysis. The present study aimed to elucidate understanding of the anti-fatigue and anti-hypoxia effects of $P$. hepiali

\section{Materials and methods}

Strain culture. Paecilomyces hepiali, purchased from Anhui Agricultural University (Anhui, China; RCEF1429), was cultured in a 100 liter full-automatic fermenter (Biotech-100JS; Baoxing Bioscience Company, Shanghai, China) at $26^{\circ} \mathrm{C}$ for 5 days using a defined liquid medium containing $25 \mathrm{~g} / 1$ sucrose, $10 \mathrm{~g} / \mathrm{l}$ peptone, $18 \mathrm{~g} / \mathrm{l}$ yeast extract powder, $3 \mathrm{~g} / 1 \mathrm{KH}_{2} \mathrm{PO}_{4}, 3 \mathrm{~g} / \mathrm{l}$ $\mathrm{MgSO}_{4} \cdot 7 \mathrm{H}_{2} \mathrm{O}, 10 \mathrm{~g} / \mathrm{l}\left(\mathrm{NH}_{4}\right)_{2} \mathrm{SO}_{4}, 0.01 \mathrm{~g} / 1 \mathrm{ZnCl}_{2}$ and $0.24 \mathrm{~g} / 1$ vitamin $\mathrm{B}_{1}$ (all obtained from Sigma-Aldrich, St. Louis, MO, USA). The mycelia were harvested by centrifugation at $2,667 \mathrm{x} \mathrm{g}$ for $10 \mathrm{~min}$ at $4^{\circ} \mathrm{C}$, and were lyophilized for further use in a Genesis SQ 25ES lyophilizer (SP Industries, Inc., Warminster, PA, USA) (6). All chemical reagents used in the submerged fermentation were obtained from Sigma-Aldrich.

Crude extract preparation. The aqueous extract from the Paecilomyces hepiali was extracted at $80^{\circ} \mathrm{C}$ for $4 \mathrm{~h}$, which was performed twice. Following centrifugation at 2,667 $\mathrm{x}$ g for $10 \mathrm{~min}$ at $4^{\circ} \mathrm{C}$, the supernatant was sequentially concentrated in an evaporator (R1002B; Shanghai SENCO Technology Co., Ltd., Shanghai, China) under reduced pressure $(0.09 \mathrm{mPa}$ at $80^{\circ} \mathrm{C}$ ), and was then freeze-dried to produce the solid aqueous extract, PHC (21).

Animal care. The experimental animal protocol used in the present study was approved by the Lab Animal Centre of Jilin University [Changchun, China; SCXK (JI)-2011-0003] and the present study was approved by the ethics committee of Jilin University. KunMing (KM) mice (6-week-old; 18-22 g,
1:1 male: female ratio, $n=20$ /group), purchased from Norman Bethune University of Medical Science, Jilin University, were maintained in a 12-h light/dark cycle (lights on 07:00-19:00) at $23 \pm 1^{\circ} \mathrm{C}$ with water and food available ad libitum. At $8 \mathrm{~h}$ prior to initiation of the experiment, the animals were deprived of food, with free access to water. All experiments were performed in a quiet room, and each animal (total, 600) was used only once.

Anti-fatigue resistance assessment. The KM mice were randomly divided into five groups ( $\mathrm{n}=20 /$ group; $1: 1$ male to female ratio), and orally administered with double distilled (D.D.) water (vehicle group), $0.6 \mathrm{~g} / \mathrm{kg}$ rhodiola capsule (positive group) (22) and PHC at doses of 0.04, 0.2 and $1.0 \mathrm{~g} / \mathrm{kg}$ once per day for 7 days. At the end of drug administration, the following experiments were performed.

Autonomic activity assessment. The mice were placed in a multichannel activity box (ZZ-6; Taimeng Science Technology, Ltd., Chengdu, China) and locomotor activities were measured for $5 \mathrm{~min}$. The use of an infrared sensor with multiple Fresnel lenses (component of ZZ-6) enabled vertical movements, including jumping, as well as horizontal movements, including walking and running, to be counted. Measurements were performed between 12:00 and 16:00 (23).

Forced running assessment. The endurance of the mice was assessed on a treadmill (FT-200; Taimeng Science Technology, Ltd.), which allowed them to run at a set speed of $20 \mathrm{mph}$ for $1 \mathrm{~min}$. Following three training exercises, the mice were placed on the treadmill at the $20 \mathrm{mph}$ speed. The number of shocks received from an electrode, touched when the mice cannot run at the set speed, in a $5 \mathrm{~min}$ period was used to evaluate running performance (23).

Rotating rod assessment. A fatigue turning device (ZB-200; Taimeng Science Technology, Ltd.) was used to determine mouse performance following PHC administration for 7 days. Prior to formal assessment, the mice were allowed three training exercises, in which a speed of $20 \mathrm{rpm}$ was applied for $1 \mathrm{~min}$. For subsequent fatigue analysis, the mice were placed on the turning device at a speed of $20 \mathrm{rpm}$, and the total duration for which the mouse remained on the rod was recorded.

Weight-loaded forced swimming assessment. Following the 7 day PHC administration, a weight-loaded forced swimming assessment was performed to evaluate the endurance and performance of each mouse, using a method described previously, with minor modifications (24). The mice were monitored swimming in water when loaded with a weight equivalent to $10 \%$ of their body weight. The temperature and depth of the water were $22 \pm 1^{\circ} \mathrm{C}$ and $30 \mathrm{~cm}$, respectively. Exhaustion duration was determined from the beginning of swimming to the point at which the mice failed to return to the water surface within $15 \sec (12)$.

Antihypoxic capacity assessment. As with the assessment of antifatigue, the KM mice were randomly divided into five groups ( $n=20 /$ group; 1:1 male: female ratio), and orally 
A

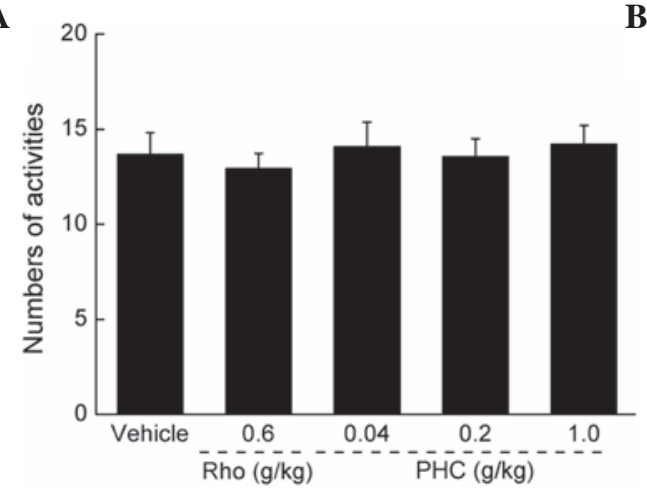

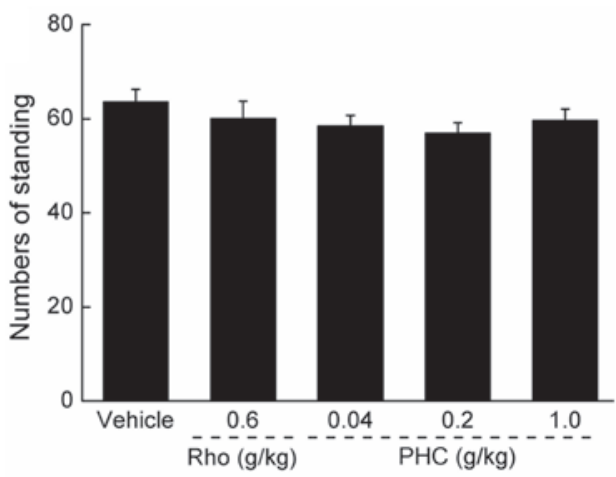

Figure 1. PHC does not affect autonomic activity in mice. The effects of PHC (0.04,0.2 and $1 \mathrm{~g} / \mathrm{kg})$ and rhodiola capsule (0.6 g/kg) on (A) mouse spontaneous locomotor activity and (B) spontaneous standing were determined following 7 days treatment, respectively. Data are expressed as the mean \pm standard deviation $(n=20)$ and analyzed using one-way analysis of variance followed by Dunn's test. PHC, Paecilomyces hepiali extract; Rho, rhodiola capsule.

administered with either D.D. water (vehicle group), $0.6 \mathrm{~g} / \mathrm{kg}$ rhodiola capsule (positive group) (22) or PHC at doses of 0.04 0.2 and $1.0 \mathrm{~g} / \mathrm{kg}$ once a day for 7 days.

Normobaric hypoxia assessment. At $60 \mathrm{~min}$ following the final administration, each mouse was placed into a $250 \mathrm{ml}$ airtight container containing medical soda lime (Sinopharm Chemical Reagent Co., Ltd., Shanghai, China). The duration of survival in oxygen deprivation was recorded.

Sodium nitrite toxicosis assessment. At $60 \mathrm{~min}$ following the final administration, each mouse was injected with $240 \mathrm{mg} / \mathrm{kg}$ sodium nitrite (Sinopharm Chemical Reagent Co., Ltd.) intraperitoneally, and the duration of survival was recorded.

Acute cerebral ischemia assessment. At $60 \mathrm{~min}$ following the final administration, each mouse was sacrificed immediately by decapitation. The duration of time between decapitation and the final gasp was recorded.

Sample collection. Following overnight fasting, the mice ( $\mathrm{n}=20 /$ group; 1:1 male: female ratio) were orally administered with either D.D. water as the vehicle group, $0.6 \mathrm{~g} / \mathrm{kg}$ rhodiola capsule as the positive group or PHC, at doses of 0.04, 0.2 and $1.0 \mathrm{~g} / \mathrm{kg}$, once a day for 7 days. At $60 \mathrm{~min}$ following the final treatment, 10 mice in each group were forced to swim for $60 \mathrm{~min}$ and recess for $10 \mathrm{~min}$, following which $0.2 \mathrm{ml}$ blood samples were collected from the caudal vein of the mice. At the end of the experiment, the mice were sacrificed by injection of $200 \mathrm{mg} / \mathrm{kg}$ pentobarbital (Beijing Chemical Reagent Company, Beijing, China) and liver tissues were dissected, washed with ice-cold physiological saline, and homogenized in D.D. water.

Parameter determination. The levels of ATP, superoxide dismutase (SOD) and glutathione peroxidase (GSH-Px) in the serum and liver tissues were determined according to the manufacturer's protocol of the associated assay kits, Superoxide Dismutase assay kit (WST-1 method) and the Glutathione Peroxidase assay kit (colorimetric method; Nanjing Jiangcheng Bioengineering Institute, Nanjing, China).
Western blot analysis. The liver tissue samples were homogenized using 5-10 volumes of lysis buffer containing $1 \mathrm{mM}$ phenylmethanesulfonyl fluoride (Sigma-Aldrich) and $1 \mathrm{X}$ protease inhibitor cocktail (Sigma-Aldrich). The homogenate was centrifuged at $9,588 \times \mathrm{g}$ for $10 \mathrm{~min}$ at $4^{\circ} \mathrm{C}$, and the resulting supernatants were used as the whole protein extract. The total protein was estimated using a Bicinchoninic Acid Assay kit (Nanjing Biotechnology Co., Ltd.), and $40 \mu \mathrm{g}$ protein was separated by $10 \%$ SDS-PAGE [30\% acrylamide (Solarbio Science and Technology Co., Ltd., Beijing, China), SDS (Sinopharm Chemical Reagent Co.,Ltd.), ammonium persulfate (Sinopharm Chemical Reagent Co., Ltd.), tetramethylethylenediamine (Beijing Dingguo Changsheng Biotechnology Co., Ltd, Beijing, China) and buffer solution] and transferred onto a nitrocellulose membrane $(0.45 \mu \mathrm{m}$; Bio Basic, Inc, Markham, ON, Canada) using an electroblotting apparatus (PowerPac $^{\mathrm{TM}}$ power supply and Mini-PROTEAN ${ }^{\circledR}$ Tetra Cell; Bio-Rad Laboratories, Inc., Hercules, CA, USA) at $100 \mathrm{~V}$ for $120 \mathrm{~min}$. The transferred membranes were then blotted with the following primary antibodies at $4^{\circ} \mathrm{C}$ overnight, at dilutions of 1:1,000: Rabbit anti-mouse monoclonal phosphorylated (p)-mTOR (Abcam, Cambridge, MA, USA; cat. no ab109268); rabbit anti-mouse polyclonal total (t)-mTOR (Abcam; cat. no. ab83495); mouse anti-mouse monoclonal p-AKT (EMD Millipore, Billerica, MA, USA; cat. no. 05-1003); rabbit anti-mouse polyclonal t-AKT (Abcam; cat. no. ab126811); rabbit anti-mouse polyclonal p-AMPK (EMD Millipore; cat. no. 07-681); rabbit anti-mouse polyclonal t-AMPK (EMD Millipore; cat. no. 07-181); and rabbit anti-mouse polyclonal glyceraldehyde-3-phosphate dehydrogenase (EMD Millipore; cat. no. ABS16). The membranes were subsequently incubated with horseradish peroxidase-conjugated mouse anti-rabbit IgG (Santa Cruz Biotechnology, Inc., Dallas, TX, USA; cat. no. sc-2357; 1:2,000) and goat anti-mouse IgG (Santa Cruz Biotechnology, Inc.; cat. no. sc-2005; 1:2,000) secondary antibodies at $4^{\circ} \mathrm{C}$ for $4 \mathrm{~h}$. Chemiluminescence was detected using an ECL detection kit (GE Healthcare Life Sciences, Chalfont, UK). The intensity of the bands was quantified by scanning densitometry using Quantity One-4.5.0 software (Bio-Rad Laboratories, Inc.).

Statistical analysis. All values are expressed as the mean \pm standard deviation. One-way analysis of variance 
A
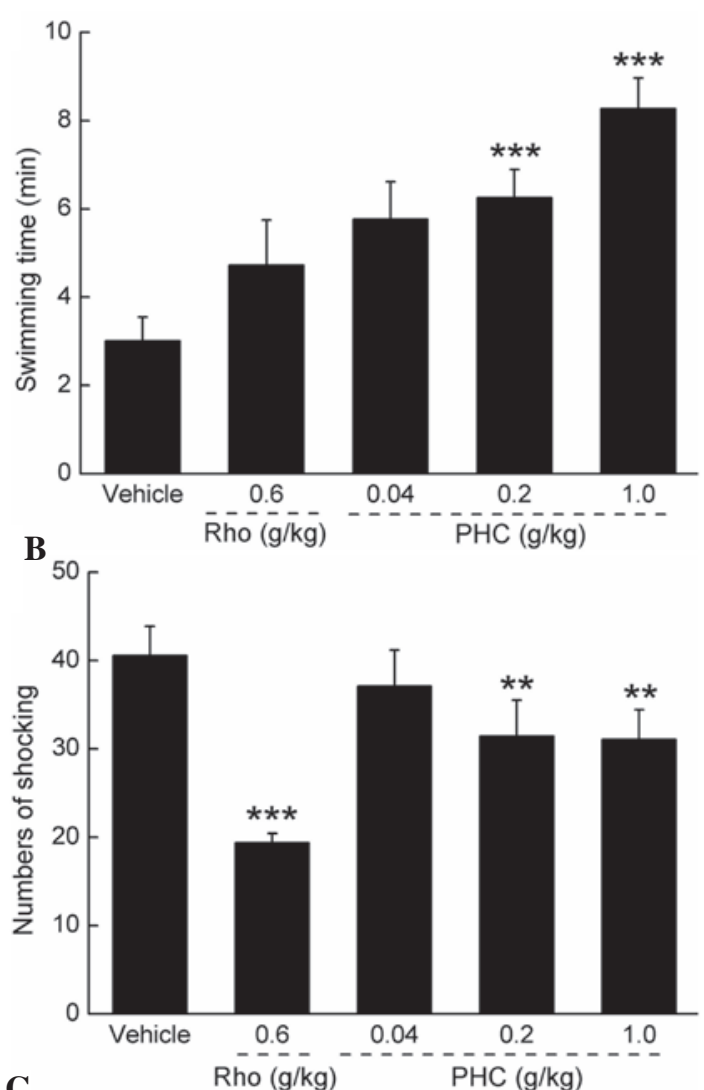

C

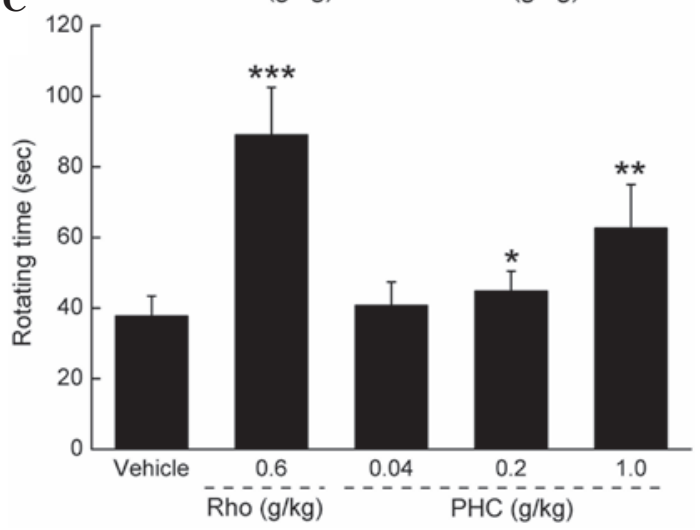

Figure 2. PHC enhances antifatigue effects. The antifatigue effects of PHC $(0.04,0.2$ and $1 \mathrm{~g} / \mathrm{kg})$ and rhodiola capsule $(0.6 \mathrm{~g} / \mathrm{kg})$ treatment were analyzed by performing a (A) forced swimming, (B) forced running and (C) rotating rod assessments. Data are expressed as the mean \pm standard deviation $(n=20)$ and analyzed using one-way analysis of variance followed by Dunn's test. ${ }^{*} \mathrm{P}<0.05,{ }^{* *} \mathrm{P}<0.01,{ }^{* * *} \mathrm{P}<0.001$ vs. vehicle-treated mice. $\mathrm{PHC}$, Paecilomyces hepiali extract; Rho, rhodiola capsule.

was use to detect statistical significance, followed by post-hoc multiple comparison using Dunn's test. Statistical analysis was conducted using SPSS 16.0 software (SPSS, Inc., Chicago, IL, USA) and $\mathrm{P}<0.05$ was considered to indicate a statistically significant difference.

\section{Results}

Effects of PHC on autonomic activity. No significant effects on mouse autonomic activity were observed following PHC treatment, indicating that $\mathrm{PHC}$ was a safe agent for use in the subsequent experiments ( $\mathrm{P}>0.05$; Fig. 1$)$.
A
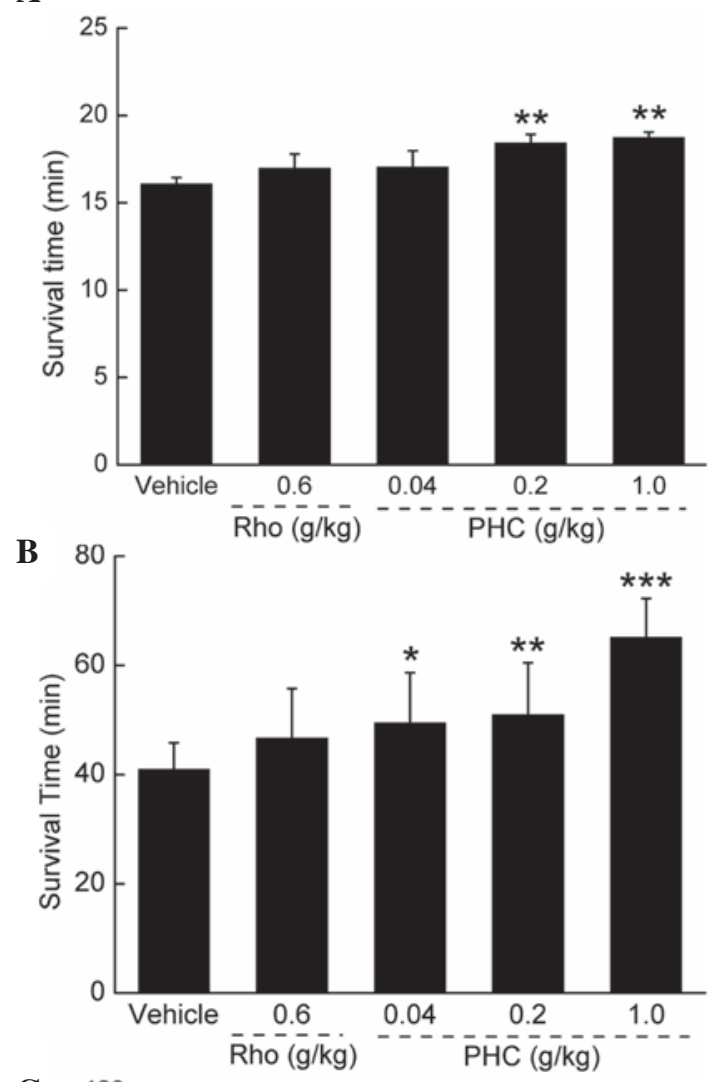

C

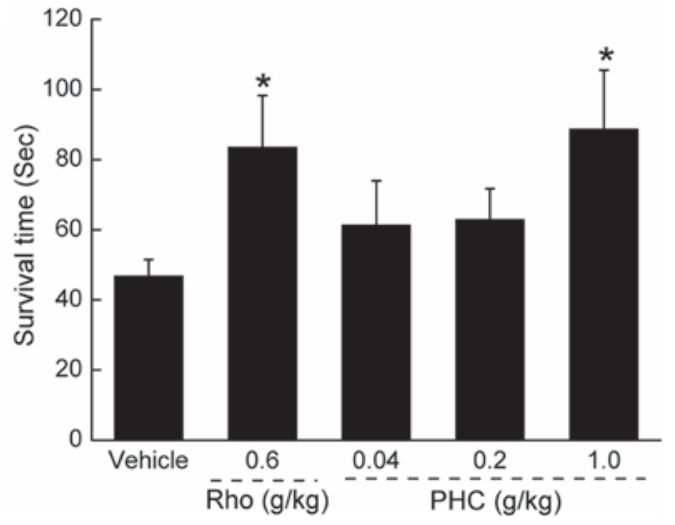

Figure 3. PHC has antihpoxic effects. The antihypoxic effects of PHC $(0.04$ 0.2 and $1 \mathrm{~g} / \mathrm{kg})$ and rhodiola capsule $(0.6 \mathrm{~g} / \mathrm{kg})$ treatment were analyzed using a (A) normobaric hypoxia, (B) sodium nitrite toxicosis and (C) acute cerebral ischemia assessment. Data are expressed as the mean \pm standard deviation $(n=20)$ and analyzed using one-way analysis of variance followed by Dunn's test. ${ }^{*} \mathrm{P}<0.05,{ }^{* *} \mathrm{P}<0.01,{ }^{* * *} \mathrm{P}<0.001$ vs. vehicle-treated mice. PHC, Paecilomyces hepiali extract; Rho, rhodiola capsule.

Antifatigue activities of PHC. The antifatigue activities of PHC were detected via forced swimming, forced running and rotating rod assessments. Similar to previous findings in Herba rhodiolae (19), PHC treatment significantly enhanced swimming duration, with a maximum recording of $8.26 \mathrm{~min}$, compared with the duration of $3.01 \mathrm{~min}$ in the control group $(\mathrm{P}<0.001$; Fig. 2A). In the forced running assessment, the number of shocks were significantly reduced following the administration of 0.2 and $1 \mathrm{~g} / \mathrm{kg}$ PHC for 7 days, compared with the control $(\mathrm{P}<0.01$; Fig. $2 \mathrm{~B})$. The duration for which the mice remained on the rotating rod were recorded to evaluate the antifatigue activities of PHC. Compared with the mice in 
A

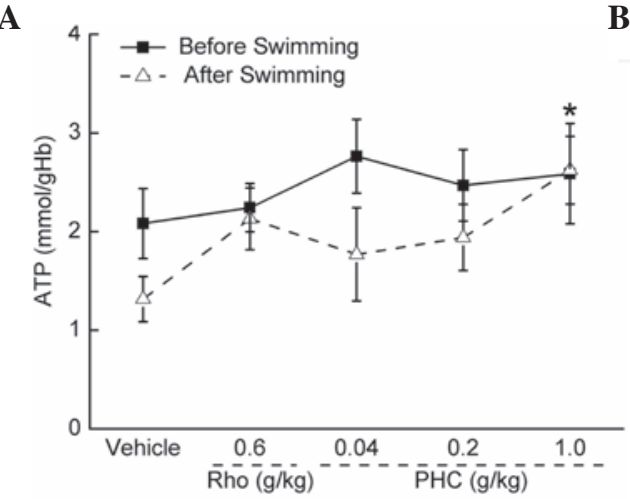

B

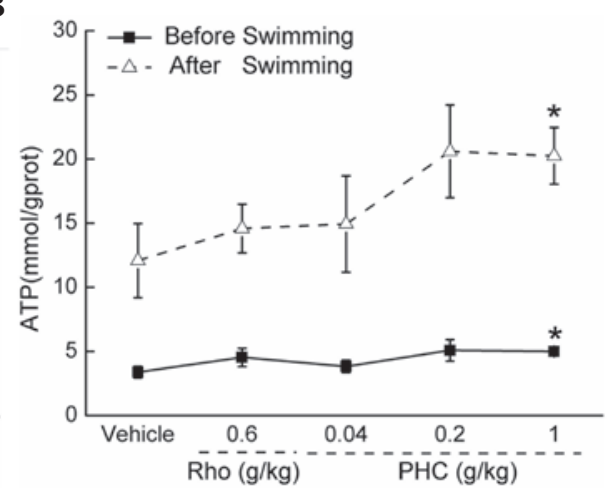

Figure 4. PHC increases levels of ATP. Following 7 days treatment with PHC $(0.04,0.2$ and $1 \mathrm{~g} / \mathrm{kg})$ or rhodiola capsule $(0.6 \mathrm{~g} / \mathrm{kg})$, the effect of PHC on ATP metabolism was analyzed. (A) Level of ATP in serum prior to and following 60-min swimming. (B) Level of ATP in the liver prior to and following 60-min swimming. Data are expressed as the mean \pm standard deviation $(\mathrm{n}=10)$ and analyzed using one-way analysis of variance followed by Dunn's test. "P<0.05, vs. vehicle-treated mice. PHC, Paecilomyces hepiali extract; Rho, rhodiola capsule; ATP, adenosine triphosphate.

A

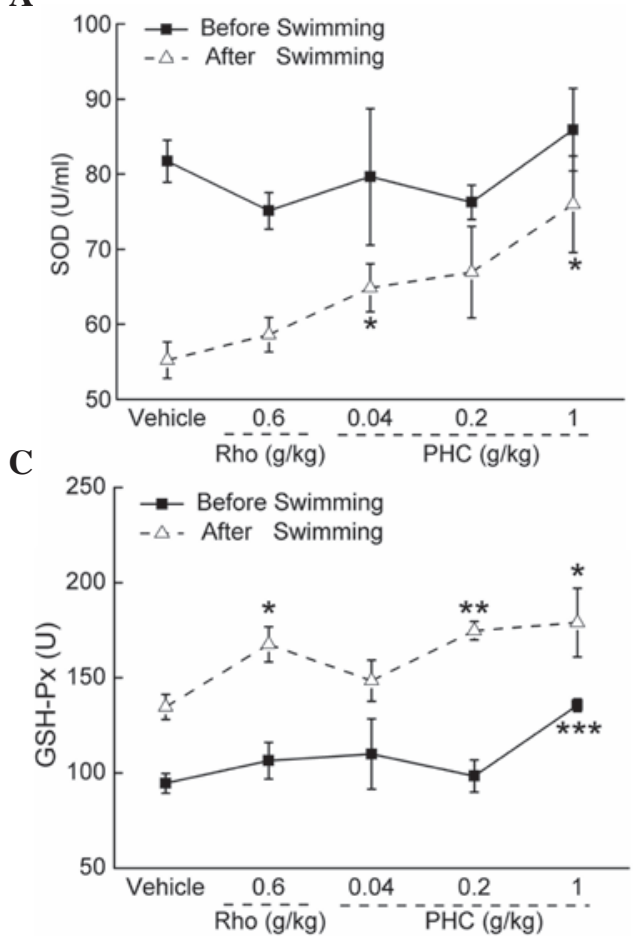

B

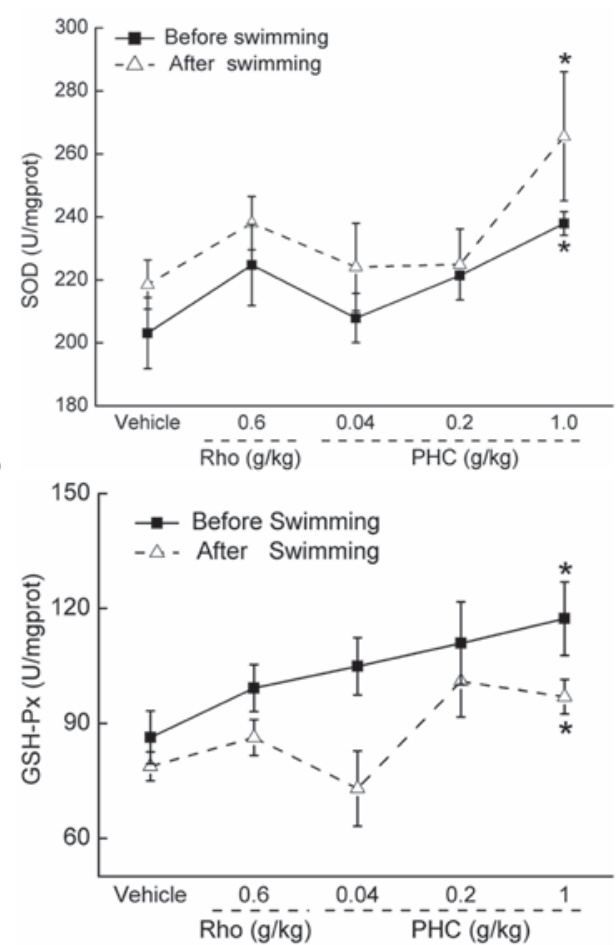

Figure 5. PHC increases levels of SOD and GSH-Px. Mice were treated with PHC $(0.04,0.2$ and $1 \mathrm{~g} / \mathrm{kg})$ or rhodiola capsule $(0.6 \mathrm{~g} / \mathrm{kg})$ for $7 \mathrm{days}$, prior to and following 60-min swimming, the activities of SOD activities in the (A) serum and (B) liver, and levels of GSH-Px in the (C) serum and (D) liver were determined, respectively. Data are expressed as the mean \pm standard deviation $(\mathrm{n}=10)$ and analyzed using one-way analysis of variance followed by Dunn's test. ${ }^{*} \mathrm{P}<0.05,{ }^{* *} \mathrm{P}<0.01$, and ${ }^{* * *} \mathrm{P}<0.001$ vs. vehicle-treated mice. PHC, Paecilomyces hepiali extract; Rho, rhodiola capsule; SOD, superoxide dismutase; GSH-Px, glutathione peroxidase.

the control group, 0.2 and $1 \mathrm{~g} / \mathrm{kg}$ PHC treatment enhanced the duration remaining on the rod by almost 18.91 and $66.17 \%$, respectively $(\mathrm{P}<0.05$; Fig. $2 \mathrm{C})$.

Antihypoxic activities of PHC. In the sodium nitrite toxicosis assessment, $1 \mathrm{~g} / \mathrm{kg}$ PHC administration extended the survival duration by $16.5 \%$, compared with the vehicle-treated mice, and by almost $10.3 \%$, compared with the rhodiola capsule $(\mathrm{P}<0.01$; Fig. 3A). In the normobaric hypoxia assessment, as with Herba rhodiolae, PHC dose-dependently increased survival duration in the mice exposed to hypoxia $(\mathrm{P}<0.05$; Fig. 3B). Compared with the control group, treatment with
$1 \mathrm{~g} / \mathrm{kg}$ PHC enhanced survival duration by almost $59.07 \%$ $(\mathrm{P}<0.001)$. Additionally, in the acute cerebral ischemia assessment, $1 \mathrm{~g} / \mathrm{kg}$ PHC and $0.6 \mathrm{~g} / \mathrm{kg}$ rhodiola capsule improved survival duration by 89.64 and $78.60 \%$, compared with the vehicle-treated and $0.6 \mathrm{~g} / \mathrm{kg}$ rhodiola capsule-treated groups $(\mathrm{P}<0.05$; Fig. 3C).

PHC increases the levels of ATP, SOD and GSH-PX in the serum and liver. Following treatment for 7 days, prior to swimming, $1 \mathrm{~g} / \mathrm{kg}$ PHC led to an increase of $99.28 \%$ in serum ATP concentration, compared with the control group $(\mathrm{P}<0.05$; Fig. 4A). A similar trend of PHC was observed following $60 \mathrm{~min}$ swimming 

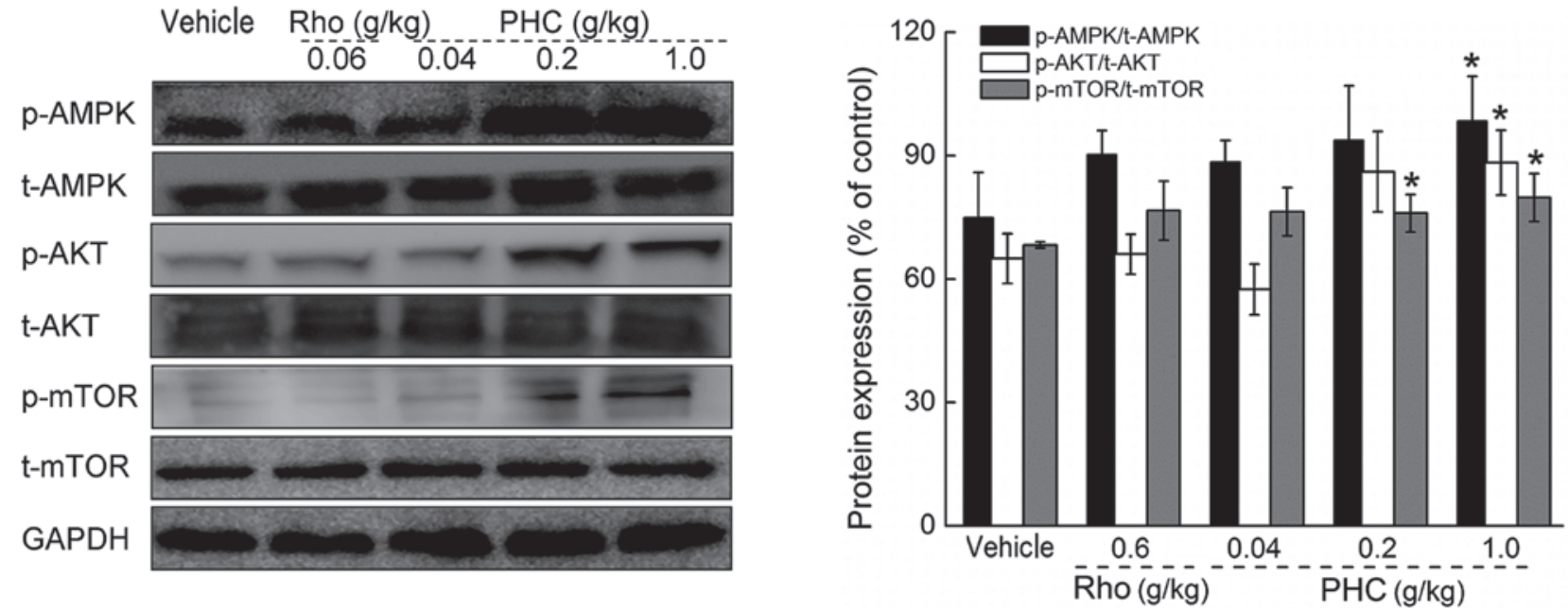

Figure 6. Mice were treated with PHC $(0.04,0.2$ and $1 \mathrm{~g} / \mathrm{kg})$ or rhodiola capsule $(0.6 \mathrm{~g} / \mathrm{kg})$ for 7 days, following 60 -min swimming, and the activation of AKT, AMPK and mTOR in the liver were analyzed using Western blot analysis. Quantification of the expression levels of p-AKT, p-AMPK and p-mTOR were normalized by corresponding levels of t-AKT, t-AMPK and t-mTOR. Data are expressed as the mean \pm standard deviation (n=10) and analyzed using one-way analysis of variance followed by Dunn's test. " $\mathrm{P}<0.05$, vs. vehicle-treated mice. PHC, Paecilomyces hepiali extract; Rho, rhodiola capsule; p-phosphorylated; t-, total; AKT, protein kinase B; AMPK, 5'-monophosphate-activated protein kinase; mTOR, mammalian target of rapamycin; GAPDH, glyceraldehyde-3-phosphate dehydrogenase.

(Fig. 4A). In the liver, the ATP concentration was significantly higher, compared with that prior to swimming. Treatment with $1 \mathrm{~g} / \mathrm{kg}$ PHC resulted in 47.45 and $67.48 \%$ increases prior to and following swimming, respectively $(\mathrm{P}<0.05$; Fig. 4B).

Treatment with $1 \mathrm{~g} / \mathrm{kg}$ PHC increased serum SOD levels by $37.58 \%$ following swimming, compared with the vehicle-treated mice $(\mathrm{P}<0.05$; Fig. 5A). Additionally, 7-day treatment with $1 \mathrm{~g} / \mathrm{kg}$ PHC enhanced the levels of SOD in the liver by 17.13 and $21.54 \%$ prior to and following swimming, respectively ( $\mathrm{P}<0.05$; Fig. 5B).

On determining the levels of GSH-Px prior to and following swimming, the same trend was noted in the serum and liver tissues. In the serum, $1 \mathrm{~g} / \mathrm{kg}$ PHC treatment resulted in increases of 43.34 and $32.94 \%$ prior to and following swimming, respectively $(\mathrm{P}<0.05$; Fig. $5 \mathrm{C})$. In the liver, increases in 35.97 and $23.08 \%$ prior to and following swimming were observed in the $1 \mathrm{~g} / \mathrm{kg}$ PHC-treated mice, respectively $(\mathrm{P}<0.05$; Fig. 5D).

Effects of PHC on the activation of $p-A K T, p-A M P K$ and $p$-mTOR. The activation of AKT, AMPK and mTOR were further analyzed in the liver tissues to investigate the underlying mechanism. In the rhodiola capsule-treated group, no significant effects on the expression levels of p-AKT, p-AMPK or p-mTOR were observed (Fig. 6). Treatment for 7 days with $1 \mathrm{~g} / \mathrm{kg}$ PHC enhanced the expression levels of p-AKT, p-AMPK and p-mTOR in the liver by $31.37,35.91$ and $16.94 \%$, compared with the vehicle-treated mice $(\mathrm{P}<0.05$; Fig. 6).

\section{Discussion}

Overexercise and acute mountain sickness, which leads to the production of increased oxygen radicals, lead to irreversible tissue damage (25). In clinical trials, various herbs have been used to alleviate the symptoms of fatigue and hypoxia $(10,22,26)$. The aim of the present study was to investigate the antifatigue and antihypoxic effects of $\mathrm{PHC}$ and examine the underlying mechanisms. Preliminary determination showed that PHC contained $27.22 \%$ polysaccharides, $20.6 \%$ total proteins, $43.06 \%$ organic acid and $1.56 \%$ adenosine.

The physiological effect of fatigue can be attributable to energy metabolism, metabolite accumulation, and muscle glycogen depletion, which are also associated with hypoxia (27). The enhancement of ATP levels in the serum and liver following 7-day administration may contribute, in part, to PHC-mediated fatigue recovery. Additionally, PHC treatment increased the levels of SOD and GSH-Px in the serum and liver, prior to and following exercise for $60 \mathrm{~min}$. SOD catalyzes the conversion of superoxide into hydrogen peroxide and oxygen; whereas GSH-Px scavenges hydroxyl radicals (28). As reported previously, antioxidant enzymes are important in preventing oxidative injury in an in vivo mice model (29). Our previous experiments confirmed that Cordyceps militaris polysaccharides upregulated the levels of SOD and GSH-Px in diabetic rats (5). Cordyceps sinensis scavenges ROS, superoxide anions and hydroxyl radicals by inhibiting malondialdehyde formation (30). In high intensity or exhaustive exercise, the overproduction of ROS is observed (31). Supporting endogenous antioxidant systems with additional oral antioxidants has been demonstrated to prevent or reduce oxidative stress, decrease muscle damage and improve exercise performance (32). The Ucp2 gene has been shown to have an antifatigue effect, efficiently improving endurance in sedentary mice, which subsequently increases the expression of antioxidant enzymes and reduces ROS levels (33). The activation of SOD and GSH leads to $\mathrm{TiO}_{2}$ removing ROS, improving the survival of $B$. mori larvae under phoxim-induced toxicity (34), alleviates fatigue (35) and enhances antihypoxic effects (36). Taken together, the regulation of oxidation-associated factors may be responsible for PHC-mediated antifatigue and antihypoxic effects. 
In the present study, PHC was also found to improve the activities of AMPK, AKT and mTOR in the mouse liver tissues following 60-min swimming. AKT phosphorylation is generally considered to enhance the activity of mTOR, which further sense cellular nutrients, oxygen and energy levels (37). AMPK is known to be important in energy homeostasis, and is considered a major switch, regulating glucose and lipid metabolism (38). In abnormal conditions, including starvation, hypoxia and oxidative stress, activated AMPK promotes cell survival (39). In the liver, AMPK switches on ATP-producing processes and inhibits ATP-consuming anabolic processes (40), and it has been reported that treatment with the AMPK agonist, 5-aminoimidazole carboxamide ribonucleotide, can induce the expression levels of metabolic genes and enhance running endurance (41). Once activated by falling cellular energy status, AMPK activates catabolic pathways, which generate ATP whilst inhibiting anabolic pathways and other cellular processes that consume ATP (42). In the PHC-treated mice in the present study, the enhanced ATP concentration in the serum and liver following 60-min swimming may have combined with AMPK phosphorylation. Furthermore, as an axis of energy metabolism, AMPK activation counteracts oxidative stress by inhibiting $\mathrm{NAD}(\mathrm{P}) \mathrm{H}$ oxidase-derived ROS accumulation (43). Via activation of the AMPK-sterol regulatory element-binding protein signaling pathway, the levels of SOD and GSH-Px in the liver are enhanced (44). The results of the present study suggested that the antifatigue and antihypoxic effects of PHC treatment were predominantly through modulation of the AMPK pathway.

A limitation of the present study was that the data did not permit investigation of the association between the AMPK and AKT/mTOR pathways. In previous investigations performed in in cancer cells or brain tissue, an increase in p-AKT and a decrease in p-AMPK has been demonstrated to lead to the increased phosphorylation of mTOR (45). However, PHC treatment enhanced the phosphorylation of AMPK and MTOR in the liver tissue. Further investigations are required to elucidate the underlying mechanism in more details.

In conclusion, the present study demonstrated that PHC induced recovery from fatigue and hypoxia in mice, at least partially via the activation of the AMPK and AKT/mTOR pathways. PHC treatment resulted in increases in the levels of ATP, SOD and GSH-Px in the serum and liver tissues. These data provide experimental evidence supporting the clinical use of PHC as an effective agent against fatigue and hypoxia.

\section{Acknowledgements}

Thisstudy was supported by theNationalScienceand Technology Support Program of P.R. China (grant no. 2012BAL29B05), the National Natural Science Foundation of P.R. China (grant no. 81402955), and the Science and Technology Key Project in Jilin Province (grant no. 20130201006ZY).

\section{References}

1. Chen PX, Wang SN, Nie SP and Marcone M: Properties of Cordyceps sinensis: A review. J Funct Foods 5: 550-569, 2013.

2. Wu Z, Yang Z, Gan D, Fan J, Dai Z, Wang X, Hu B, Ye H, Abid M and Zeng $X$ : Influences of carbon sources on the biomass, production and compositions of exopolysaccharides from Paecilomyces hepiali HN1. Biomass Bioenergy 67: 260-269, 2014.
3. Yu SJ, Zhang Y, Li CR, Zhang Q, Ma ZY and Fan MZ: Optimization of ultrasonic extraction of mycelial polysaccharides from Paecilomyces hepiali using response surface methodology and its antioxidant activity. African Journal of Biotechnology 10: 17241-17250, 2011.

4. Thakur A, Hui R, Hongyan Z, Tian Y, Tianjun C and Mingwei C: Pro-apoptotic effects of Paecilomyces hepiali, a Cordyceps sinensis extract on human lung adenocarcinoma A549 cells in vitro. J Cancer Res Ther 7: 421-426, 2011.

5. Dong Y, Jing T, Meng Q, Liu C, Hu S, Ma Y, Liu Y, Lu J, Cheng Y, Wang D and Teng L: Studies on the antidiabetic activities of Cordyceps militaris extract in diet-streptozotocin-induced diabetic Sprague-Dawley rats. Biomed Res Int 2014: 160980, 2014.

6. Dong Y, Hu S, Liu C, Meng Q, Song J, Lu J, Cheng Y, Gao C, Liu Y, Wang D and Teng L: Purification of polysaccharides from Cordyceps militaris and their anti-hypoxic effect. Mol Med Rep 11: 1312-1317, 2015.

7. Zhang W, Wu SZ, Cao JL, Li HM, Li Y, He JG and Zhang LB: A preliminary study on anti-hypoxia activity of yak milk powder in vivo. Dairy Science \& Technology 94: 633-639, 2014.

8. You L, Ren J, Yang B, Regenstein J and Zhao M: Antifatigue activities of loach protein hydrolysates with different antioxidant activities. J Agric Food Chem 60: 12324-12331, 2012.

9. Xu C, Lv J, Lo YM, Cui SW, Hu X and Fan M: Effects of oat $\beta$-glucan on endurance exercise and its anti-fatigue properties in trained rats. Carbohydr Polym 92: 1159-1165, 2013.

10. Xie Y, Jiang S, Su D, Pi N, Ma C and Gao P: Composition analysis and anti-hypoxia activity of polysaccharide from Brassica rapa L. Int J Biol Macromol 47: 528-533, 2010.

11. Zou D, Chen K, Liu P, Chang H,Zhu J and Mi M: Dihydromyricetin improves physical performance under simulated high altitude. Med Sci Sports Exerc 46: 2077-2084, 2014.

12. Wu RM, Sun YY, Zhou TT, Zhu ZY, Zhuang JJ, Tang X, Chen J, $\mathrm{Hu} \mathrm{LH}$ and Shen X: Arctigenin enhances swimming endurance of sedentary rats partially by regulation of antioxidant pathways. Acta Pharmacol Sin 35: 1274-1284, 2014.

13. Bijland S, Mancini SJ and Salt IP: Role of AMP-activated protein kinase in adipose tissue metabolism and inflammation. Clin Sci (Lond) 124: 491-507, 2013.

14. Dekany M, Nemeskéri V, Györe I, Harbula I, Malomsoki J and Pucsok J: Antioxidant status of interval-trained athletes in various sports. Int J Sports Med 27: 112-116, 2006

15. Bogdanis GC, Stavrinou P, Fatouros IG, Philippou A, Chatzinikolaou A, Draganidis D, Ermidis G and Maridaki M: Short-term high-intensity interval exercise training attenuates oxidative stress responses and improves antioxidant status in healthy humans. Food Chem Toxicol 61: 171-177, 2013.

16. Pandareesh MD and Anand T: Ergogenic effect of dietary L-carnitine and fat supplementation against exercise induced physical fatigue in Wistar rats. J Physiol Biochem 69: 799-809, 2013.

17. Gao H, Long Y, Jiang X, Liu Z, Wang D, Zhao Y, Li D and Sun BL: Beneficial effects of Yerba Mate tea (Ilex paraguariensis) on hyperlipidemia in high-fat-fed hamsters. Exp Gerontol 48: 572-578, 2013.

18. Ni W, Gao T, Wang H, Du Y,Li J,Li C, Wei Land Bi H: Anti-fatigue activity of polysaccharides from the fruits of four Tibetan plateau indigenous medicinal plants. J Ethnopharmacol 150: 529-535, 2013.

19. Liu X, Zhu W, Guan S, Feng R, Zhang H, Liu Q, Sun P, Lin D, Zhang $\mathrm{N}$ and Shen J: Metabolomic analysis of anti-hypoxia and anti-anxiety effects of Fu Fang Jin Jing Oral Liquid. PLoS One 8: e78281, 2013

20. Panossian A and Wagner H: Stimulating effect of adaptogens: An overview with particular reference to their efficacy following single dose administration. Phytother Res 19: 819-838, 2005.

21. Du LN, Song J, Wang HB, Li P, Yang ZZ, Meng LJ, Meng FQ, Lu JH and Teng LR: Optimization of the fermentation medium for Paecilomyces tenuipes N45 using statistical approach. African Journal of Microbiology Research 6: 6130-6141, 2012.

22. Zhang CX and Dai ZR: Anti-hypoxia activity of a polysaccharide extracted from the Sipunculus nudus L. Int J Biol Macromol 49: 523-536, 2011

23. Nakagawasai O, Yamada K, Nemoto W, Fukahori M, Tadano T and Tan-No K: Liver hydrolysate assists in the recovery from physical fatigue in a mouse model. J Pharmacol Sci 123: 328-335, 2013. 
24. Nallamuthu I, Tamatam A and Khanum F: Effect of hydroalcoholic extract of Aegle marmelos fruit on radical scavenging activity and exercise-endurance capacity in mice. Pharm Biol 52: 551-559, 2014.

25. Wu C, Chen R, Wang XS, Shen B, Yue W and Wu Q: Antioxidant and anti-fatigue activities of phenolic extract from the seed coat of Euryale ferox Salisb. and identification of three phenolic compounds by LC-ESI-MS/MS. Molecules 18: 11003-11021, 2013.

26. Cai Y, Lu Y, Chen R, Wei Q and Lu X: Anti-hypoxia activity and related components of Rhodobryum giganteum par. Phytomedicine 18: 224-229, 2011.

27. Bowtell JL, Cooke K, Turner R, Mileva KN and Sumners DP: Acute physiological and performance responses to repeated sprints in varying degrees of hypoxia. J Sci Med Sport 17: 399-403, 2014.

28. Borges P, Oliveira B, Casal S, Dias J, Conceição L and Valente L: Dietary lipid level affects growth performance and nutrient utilisation of Senegalese sole (Solea senegalensis) juveniles. Br J Nutr 102: 1007-1014, 2009.

29. Virmani A, Gaetani F, Imam S, Binienda Z and Ali S: The protective role of L-carnitine against neurotoxicity evoked by drug of abuse, methamphetamine, could be related to mitochondrial dysfunction. Ann NY Acad Sci 965: 225-232, 2002.

30. Liu Y, E Q, Zuo J, Tao Y and Liu W: Protective effect of Cordyceps polysaccharide on hydrogen peroxide-induced mitochondrial dysfunction in HL-7702 cells. Mol Med Rep 7: 747-754, 2013.

31. Sureda A, Ferrer MD, Tauler P, Romaguera D, Drobnic F, Pujol P, Tur JA and Pons A: Effects of exercise intensity on lymphocyte $\mathrm{H} 2 \mathrm{O} 2$ production and antioxidant defences in soccer players. $\mathrm{Br}$ J Sports Med 43: 186-190, 2009.

32. Peternelj TT and Coombes JS: Antioxidant supplementation during exercise training: Beneficial or detrimental? Sports Med 41: 1043-1069, 2011.

33. Lortz S, Gurgul-Convey E, Naujok $\mathrm{O}$ and Lenzen S: Overexpression of the antioxidant enzyme catalase does not interfere with the glucose responsiveness of insulin-secreting INS-1E cells and rat islets. Diabetologia 56: 774-782, 2013.

34. Su J, Li B, Cheng S, Zhu Z, Sang X, Gui S, Xie Y, Sun Q, Cheng Z, Cheng J, et al: Phoxim-induced damages of Bombyx mori larval midgut and titanium dioxide nanoparticles protective role under phoxim-induced toxicity. Environ Toxicol 29: 1355-1366, 2014.
35. Wang X, Xing R, Chen Z, Yu H, Li R and Li P: Effect and mechanism of mackerel (Pneumatophorus japonicus) peptides for anti-fatigue. Food Funct 5: 2113-2119, 2014

36. Zhou TB, Ou C, Rong L and Drummen GP: Effect of all-trans retinoic acid treatment on prohibitin and renin-angiotensin-aldosterone system expression in hypoxia-induced renal tubular epithelial cell injury. J Renin Angiotensin Aldosterone Syst 15: 243-249, 2014.

37. Tokunaga $\mathrm{C}$, Yoshino $\mathrm{K}$ and Yonezawa K: mTOR integrates amino acid- and energy-sensing pathways. Biochem Biophys Res Commun 313: 443-446, 2004.

38. Ceddia RB: The role of AMP-activated protein kinase in regulating white adipose tissue metabolism. Mol Cell Endocrinol 366: 194-203, 2013.

39. Bonini MG and Gantner BN: The multifaceted activities of AMPK in tumor progression-why the 'one size fits all' definition does not fit at all? IUBMB Life 65: 889-896, 2013.

40. Grahame Hardie D: AMP-activated protein kinase: A key regulator of energy balance with many roles in human disease. J Intern Med 276: 543-559, 2014.

41. Narkar VA, Downes M, Yu RT, Embler E, Wang YX, Banayo E, Mihaylova MM, Nelson MC, Zou Y, Juguilon H, et al: AMPK and PPARdelta agonists are exercise mimetics. Cell 134: 405-415, 2008

42. Rios M, Foretz M, Viollet B, Prieto A, Fraga M, García-Caballero T, Costoya JA and Senaris R: Lipoprotein internalisation induced by oncogenic AMPK activation is essential to maintain glioblastoma cell growth. Eur J Cancer 50: 3187-3197, 2014

43. St-Pierre J, Drori S, Uldry M, Silvaggi JM, Rhee J, Jäger S, Handschin C, Zheng K, Lin J, Yang W, et al: Suppression of reactive oxygen species and neurodegeneration by the PGC-1 transcriptional coactivators. Cell 127: 397-408, 2006.

44. Lee HI, Yun KW, Seo KI, Kim MJ and Lee MK: Scopoletin prevents alcohol-induced hepatic lipid accumulation by modulating the AMPK-SREBP pathway in diet-induced obese mice. Metabolism 63: 593-601, 2014.

45. Russo E, Andreozzi F, Iuliano R, Dattilo V, Procopio T, Fiume G, Mimmi S, Perrotti N, Citraro R, Sesti G, et al: Early molecular and behavioral response to lipopolysaccharide in the WAG/Rij rat model of absence epilepsy and depressive-like behavior involves interplay between AMPK, AKT/mTOR pathways and neuroinflammatory cytokine release. Brain Behav Immun 42: 157-168, 2014. 